\title{
Does concomitant laparoscopic sleeve gastrectomy and hiatal hernia repair increase morbidity?
}

\author{
Mürşit Dincer ${ }^{1}$, Fadlı Doğan²
}

\author{
${ }^{1}$ Department of General Surgery, Istanbul Haseki Training and Research Hospital, \\ Istanbul, Poland \\ ${ }^{2}$ Department of General Surgery, Medical Park Hospital, Elazığ, Turkey
}

Submitted: 30 December 2017

Accepted: 31 January 2018

Arch Med Sci Civil Dis 2018; 3: e18-e20

DOI: https://doi.org/10.5114/amscd.2018.73855

Copyright @ 2018 Termedia \& Banach

\section{Abstract}

Introduction: Obesity not only leads to susceptibility to gastroesophageal reflux disease but also is itself an important independent risk factor for the development of hiatal hernia. A coexisting hiatal hernia is found in half of obese patients with gastroesophageal reflux disease symptoms. Sleeve gastrectomy is the most commonly used restrictive method in obesity surgery. In obese patients who are scheduled to undergo sleeve gastrectomy (SG) and have a hiatal hernia, hiatal hernia repair (HHR) can be combined with SG.

Material and methods: Patients who underwent SG due to morbid obesity were retrospectively evaluated. Patients who had a body mass index of $>40 \mathrm{~kg} /$ $\mathrm{m}^{2}$ and who were diagnosed with hiatal hernia by preoperative endoscopy or intraoperatively were included in the study.

Results: A total of 30 patients who underwent SG and hiatal hernia repair were included in the study. Six patients were diagnosed with hiatal hernia by preoperative endoscopy. Twenty-four patients were intraoperatively diagnosed with hiatal hernia. The hiatal defect was repaired by suturing both anteriorly and posteriorly. There were no intraoperative or postoperative complications in any patient. The mean hospital length of stay was 3.46 days (range: 3-6). The mean follow-up time was 11.9 months (range: 4-21). Thirteen patients received medication due to acid reflux symptoms in the preoperative period. Twelve patients received no medication after surgery. One patient received low-dose medication.

Conclusions: In bariatric surgery patients with hiatal hernia, HHR should also be performed. Sleeve gastrectomy + HHR is a safe and effective method for morbidly obese patients with hiatal hernia.

Key words: bariatric surgery, outcome, concomitant laparoscopic sleeve gastrectomy and hiatal hernia.

\section{Introduction}

Obesity is one of the most important health problems in developed and developing countries [1-3]. Morbid obesity is defined as a body mass index (BMI) of more than $40 \mathrm{~kg} / \mathrm{m}^{2}$. Obesity increases the risk of systemic diseases such as diabetes mellitus (DM), cardiovascular diseases, and obstructive sleep apnea, and is associated with a high mortality rate. Obesity is also an independent risk factor for gastroesophageal reflux disease. Obesity not only leads to susceptibility to gastroesophageal reflux disease but also is itself an important independent risk factor for

\author{
Corresponding author: \\ Dr. Mürşit Dincer \\ Department \\ of General Surgery \\ Istanbul Haseki \\ Training and \\ Research Hospital \\ Istanbul, Poland \\ Millet St Aksaray Fatih \\ 34100 Istanbul, Turkey \\ Phone: +90 5446422820 \\ E-mail: drmursitdincer@ \\ gmail.com
}


the development of hiatal hernia [4-8]. A coexisting hiatal hernia is found in half of obese patients with gastroesophageal reflux disease symptoms. Sleeve gastrectomy (SG) is the most commonly used restrictive method in obesity surgery. In obese patients who are scheduled to undergo SG and have a hiatal hernia, hiatal hernia repair (HHR) can be combined with SG [9-11]. Here we present the short-term results of patients who underwent SG and HHR.

\section{Material and methods}

Patients who underwent sleeve gastrectomy (SG) due to morbid obesity between March 2016 and August 2017 were retrospectively evaluated. Patients who had a BMI of $>40 \mathrm{~kg} / \mathrm{m}^{2}$ and who were diagnosed with hiatal hernia by preoperative endoscopy or intraoperatively were included in the study.

\section{Results}

As a result of the retrospective evaluation, a total of 30 patients who underwent SG and HHR were included in the study. Of the patients included in the study, 26 were female and 4 were male. The mean age of the patients was 42.5 years (range: 26-65). The mean BMI of the patients was $42 \mathrm{~kg}$ / $\mathrm{m}^{2}$. Six patients were diagnosed with hiatal hernia by preoperative endoscopy. Twenty-four patients were intraoperatively diagnosed with hiatal hernia. During SG, the gastric fundus was completely released, and the left crus was routinely exposed. In all patients, a $37 \mathrm{Fr}$ bougie was used as standard. After SG, HHR was performed in the patients who were known to have a hiatal hernia or were intraoperatively diagnosed with hiatal hernia during exploration. During repair, 3/0 polypropylene suture material was routinely used. The hiatal defect was repaired by suturing both anteriorly and posteriorly. The prosthetic material was not used in the patients undergoing HHR. There were no intraoperative or postoperative complications in any patient. The mean hospital length of stay was 3.46 days (range: 3-6). The mean follow-up time was 11.9 months (range: 4-21). Thirteen patients received medication due to acid reflux symptoms in the preoperative period. Twelve patients received no medication after surgery. One patient received low-dose medication.

\section{Discussion}

Obesity is one of the most important diseases of our time. The prevalence of obesity has been increasing in the last three decades. The prevalence of overweight and obesity has increased to $27.5 \%$ in adults worldwide. Many factors such as bad eating habits, high-calorie food consumption, and decreased physical activity are being blamed for this situation. Obesity causes serious comorbidity and mortality. Gastroesophageal reflux disease symptoms are commonly seen in morbidly obese patients. The severity of obesity and gastroesophageal reflux disease symptoms causes serious deterioration in the quality of life. A hiatal hernia is present in approximately half of obese patients $[5,12]$. Hiatal hernia not only exacerbates reflux symptoms but also may lead to the incomplete removal of the gastric fundus during SG [3].

In a study of Dutta et al., it was observed that reflux symptoms, endoscopically and histologically detected gastritis, and endoscopically detected hiatal hernia were more frequent in morbidly obese patients [8]. In two different studies by Pandolfino et al. and Lee et al., it was reported that increased intragastric pressure and gastroesophageal pressure gradients increased the risk of hiatal hernia in direct proportion to increased BMI $[13,14]$.

Sleeve gastrectomy was initially performed as the first step of the two-stage duodenal switch operation in high-risk super-obese patients. Today, laparoscopic SG alone has become a standard procedure in the surgical treatment of morbid obesity [12]. However, the effect of SG on gastroesophageal reflux disease is still not fully revealed [15]. It has been thought that the decrease in reflux symptoms following SG may be due to various factors such as gastric volume reduction, faster gastric emptying, decreased intra-abdominal pressure, and reduced gastric acid production. The stomach in the shape of a tube and the presence of hiatal hernia are responsible for worsening of reflux symptoms [10]. There are studies in the literature reporting that reflux symptoms got worse after surgery in patients not treated for an existing hiatal hernia during SG [16]. In a study of Daes et al., it was found that hiatal hernia was intraoperatively detected in $25 \%$ of patients undergoing SG and that reflux symptoms decreased significantly after HHR [17]. In our study, this rate was found to be $80 \%$. It was thought that hiatal hernias were ignored and overlooked because endoscopy was performed by different endoscopists at other centers in all patients and therefore these were not reported. There are many articles in the literature stating that reflux symptoms decreased after HHR. However, Santonicola et al. reported that there was no improvement in reflux symptoms [18]. In the light of this information, it is recommended that it be repaired if the presence of a hiatal hernia is known prior to bariatric surgery or if a hiatal hernia is detected during the operation $[15,19,20]$. Failed hiatal hernia repair may lead to pouch malformations and exacerbation of reflux symptoms after surgery. There are authors advocating that sutures be placed anteriorly and posteriorly in HHR in order to prevent anterior hiatal dilatation during weight loss [11]. 
There are also articles in the literature reporting that HHR was performed safely with a mesh after SG [21, 22]. In our study, no prosthetic material was used in any patient.

Sleeve gastrectomy + hiatal hernia repair (SG + $\mathrm{HHR}$ ) has been shown to be a safe and effective surgical procedure in this study. It was revealed that dyspeptic symptoms and reflux symptoms regressed after HHR and that proton pump inhibitor usage declined. Our study has some limitations. Firstly, it was retrospectively conducted in a small number of patients. Secondly, the long-term results were unknown. Finally, the patients were called for a check-up but were not monitored by a standard follow-up protocol. The long-term results of SG + $\mathrm{HHR}$ can be obtained more reliably by prospective studies with a larger number of cases.

In conclusion, optimal weight loss and minimal morbidity are closely related to optimal management of hiatal hernia as well as the restrictive pouch in morbidly obese patients with hiatal hernia. In bariatric surgery patients with hiatal hernia, HHR should also be performed. The SG + HHR is a safe and effective method for morbidly obese patients with hiatal hernia.

\section{Conflict of interest}

The authors declare no conflict of interest.

\section{References}

1. Nocon M, Labenz J, Willich SN. Lifestyle factors and symptoms of gastro-oesophageal reflux: a population-based study. Aliment Pharmacol Ther 2006; 23 : 169-74.

2. Kang JH, Le QA. Effectiveness of bariatric surgical procedures. A systematic review and network meta-analysis of randomized controlled trials. Medicine 2017; 96: e8632.

3. Stenard F, lannelli A. Laparoscopic sleeve gastrectomy and gastroesophageal reflux. World J Gastroenterol 2015; 21: 10348-57.

4. Chang VW, Langa KM, Weir D, Iwashyna TJ. The obesity paradox and incident cardiovascular disease: a population-based study. PLoS One 2017; 12: e0188636.

5. Ng M, Fleming T, Robinson M, Thomson B, Graetz N, Margono C. Global, regional and national prevalence of overweight and obesity in children and adults 19802013: a systematic analysis. Lancet 2014; 384: 766-81.

6. Kasotakis G, Mittal SK, Sudan R. Combined treatment of symptomatic massive paraesophageal hernia in the morbidly obese. JSLS 2011; 15: 188-92.

7. Wilson LJ, Ma W, Hirschowitz BI. Association of obesity with hiatal hernia and esophagitis. Am J Gastroenterol 1999; 94: 2840-44.

8. Dutta SK, Arora M, Kireet A, Bashandy H, Gandsas A. Upper gastrointestinal symptoms and associated disorders in morbidly obese patients: a prospective study. Dig Dis Sci 2009; 54: 1243-6.

9. Crowell MD, Cheskin LJ, Musial F. Prevalence of gastrointestinal symptoms in obese and normal weight binge eaters. Am J Gastroenterol 1994; 89: 387-91.
10. Garg H, Vigneshwaran B, Aggarwal S, Ahuja V. Impact of concomitant laparoscopic sleeve gastrectomy and hiatal hernia repair on gastro-oesophageal reflux disease in morbidly obese patients. J Minim Access Surg 2017; 13: 103-8.

11. Jossart GH. Routine hiatal hernia repair to prevent GERD after sleeve gastrectomy. Bariatric Times 2011; 8 (5 Suppl): 15-6.

12. Prachand VN, Alverdy JC. Gastroesophageal reflux disease and severe obesity: fundoplication or bariatric surgery? World J Gastroenterol 2010; 16: 3757-61.

13. Pandolfino JE, El-Serag HB, Zhang Q, Shah N, Ghosh SK, Kahrilas PJ. Obesity: a challenge to esophagogastric junction integrity. Gastroenterology 2006; 130: 639-49.

14. Lee SW, Lien HC, Chang CS, Peng YC, Ko CW, Chou MC. Impact of body mass index and gender on quality of life in patients with gastroesophageal reflux disease. World J Gastroenterol 2012; 18: 5090-5.

15. Rebecchi F, Allaix ME, Patti MG, Schlottmann F, Morino M. Gastroesophageal reflux disease and morbid obesity: to sleeve or not to sleeve? World I Gastroenterol 2017; 23: 2269-75.

16. Himpens J, Dapri G, Cadiere G. A prospective randomized study between laparoscopic gastric banding and laparoscopic isolated sleeve gastrectomy: results after 1 and 3 years. Obes Surg 2006; 16: 1450-6.

17. Daes J, Jimenez M, Said N, Daza J, Dennis R. Laparoscopic sleeve gastrectomy: symptoms of gastroesophageal reflux can be reduced by changes in surgical technique. Obes Surg 2012; 22: 1874-9.

18. Santonicola A, Angrisani L, Cutolo P, Formisano G, lovino $P$. The effect of laparoscopic sleeve gastrectomy with or without hiatal hernia repair on gastroesophageal reflux disease in obese patients. Surg Obes Relat Dis 2014; 10: 250-5.

19. Rosenthal RJ, Diaz AA, Arvidsson D, et al. International Sleeve Gastrectomy Expert Panel. International sleeve gastrectomy expert panel consensus statement: best practice guidelines based on experience of $>12,000$ cases. Surg Obes Relat Dis 2012; 8: 8-19.

20. Gibson SC, Le Page PA, Taylor CJ. Laparoscopic sleeve gastrectomy: review of 500 cases in single surgeon Australian practice. ANZ J Surg 2015; 85: 673-7.

21. Korwar V, Peters M, Adjepong S, Sigurdsson A. Laparoscopic hiatus hernia repair and simultaneous sleeve gastrectomy: a novel approach in the treatment of gastroesophageal reflux disease associated with morbid obesity. J Laparoscopic Adv Surg Tech 2009; 19: 761-3.

22. Clapp B. Prosthetic bioabsorbable mesh for hiatal hernia repair during sleeve gastrectomy. JSLS 2013; 17: 641-4. 